\title{
Mechanisms for B-Cell Formation in Response to Insulin Resistance
}

\author{
a report by \\ Tomoaki Morioka, MD, PhD and Rohit N Kulkarni, MD, PhD \\ Joslin Diabetes Center and Department of Medicine, Harvard Medical School, Boston
}

DOI: 10.17925/USE.2006.00.1.2i

Changes in lifestyle and eating behavior in humans over the last century have contributed significantly to the epidemic of obesity and an associated dramatic increase in the incidence of diabetes worldwide (Zimmet, Alberti et al. 2001). Type 2 diabetes accounts for more than $90 \%$ of all diabetes cases, and is characterized by impaired insulin action in peripheral tissues such as skeletal muscle, adipose tissue, and liver (insulin resistance) and insufficient secretion of insulin from pancreatic B-cells in response to a rise in glucose. Defects in insulin action always precede the development of overt diabetes, and although increased insulin secretion initially compensates for the insulin resistance, overt diabetes occurs when B-cell compensation fails (Kahn 1998).

Obese individuals who do not develop diabetes show an increase in B-cell mass that seems to compensate for the metabolic demand and insulin resistance, while a failure of B-cell adaptation in some obese individuals triggers the development of overt type 2 diabetes. Understanding how B-cells compensate for insulin resistance will be important in the planning of therapeutic approaches to prevent and/or delay the onset of type 2 diabetes.

Adult B-cell mass can adapt to metabolic demands such as pregnancy, obesity and insulin resistance through developing islet hyperplasia and hyperinsulinemia. The mechanisms that maintain adult B-cell mass are currently intense area of research in type 2 diabetes and also in stem cell biology. Several mechanisms have been proposed to regulate adult B-cell mass including B-cell replication, B-cell size, neogenesis from duct cells and apoptosis (Kulkarni 2005) (Rhodes 2005). Among these mechanisms, studies in rodents including lineage analysis methods support B-cell replication as a major pathway for the renewal of adult B-cells (Dor, Brown et al. 2004). Two independent reports have examined the role of cyclin D2 and provide additional support for the replication hypothesis. Mice lacking cyclin D2 showed a selective decrease in B-cell expansion while maintaining a normal complement of ductal cells suggesting that the cell cycle protein is important for proliferation of B-cells independent of contributions from duct cells. Conversely, many studies have demonstrated that islet progenitor cells reside in the pancreatic ducts of adult humans and rodents, and neogenesis of B-cells from pancreatic duct cells may be one of the mechanisms of $\mathrm{B}$-cell regeneration in humans (Bonner-Weir, Taneja et al. 2000). Several factors, such as TGF-B, epidermal growth factor (EGF), extracellular matrix, and glucagon-like peptide-1 (GLP-1), have all been suggested to be involved in this proliferation process. While most of these studies have been reported to occur in rodent models of pancreatectomy or pancreatic-duct ligated models, or in vitro experimental systems, it is currently unclear whether similar mechanisms and pathways are also operative in vivo in insulin resistant states in humans.

Another potential mechanism that can contribute to B-cell expansion is mostly associated either with early tissue development or neoplasia and is termed epithelial-to-mesenchymal transition (EMT). EMT generally occurs in epithelial cells and involves disappearance of differentiated junctions, reorganization of cytoskeleton and redistribution of organelles, together transforming epithelial into mesenchymal cells (Savagner 2001). The mesenchymal cells in turn may regain a fully differentiated epithelial phenotype via a mesenchyme-to-epithelial transition (MET) or reverse EMT. It is possible that a similar process is also occurring in normal cells responding to physiological demands that require cell or tissue expansion. Indeed, features suggestive of EMT were shown to occur in vivo in mouse models of insulin resistance manifesting robust islet hyperplasia, namely - the insulin receptor (IR)/IR substrate-1 (IRS-1) double heterozygous (IR/IRS-1) mouse and the liver-specific IR knockout (LIRKO) mouse, (Kulkarni, Jhala et al. 2004) (Bruning, Winnay, et al 1997)(Kulkarni, Almind, et al, 2003). Evidence that EMT can occur in human islet precursor cells (Gershengorn, Hardikar et al. 2004), suggests this process may be a conserved response across species. It is worth exploring whether such a process occurs in early stages of diabetes in humans to allow for a compensatory islet hyperplastic response that prevents/delays the overt manifestation of the disease. 
Several hormones and nutrients that utilize varied mechanisms and signaling pathways have been implicated in islet hyperplastic processes to overcome hyperinsulinemia caused by whole body insulin resistance. A role for glucagon-like-peptide-1 7-36 (GLP-1) is supported by human and rodent studies (Stoffers 2004). GLP-1 receptor agonists, among which exendin-4 has been developed for clinical use, not only acutely lowers blood glucose but also engages signaling pathways in the B-cell that lead to stimulation of $\mathrm{B}$-cell replication and neogenesis and inhibition of B-cell apoptosis (Brubaker and Drucker 2004). Through a similar mechanism, glucosedependent insulinotropic polypeptide (GIP) receptor activation stimulates insulin secretion, enhances B-cell proliferation, and reduces apoptosis (Wideman and Kieffer 2004). Moreover, potentiation of the endogenous postprandial GLP-1 and GIP levels by inhibition of dipeptidyl peptidase-IV (DPP-IV) also expands B-cell mass through related mechanisms (Pospisilik, Martin et al. 2003).

Recent reports describe a fraction of patients who have undergone gastric by-pass surgery manifest severe hypoglycemia and hyperinsuliemia that is inappropriate for the ambient glycemia. While most of these patients exhibit altered B-cell mass, some of them show a greater than normal GLP-1 secretory response after a meal suggesting the incretin hormone may contribute to either an increase in B-cell mass and/or insulin hypersecretion. It is worth noting that almost all patients manifest hyperinsuliemia that is inappropriate for the levels of circulating glucose, prompting the notion that insulin itself is a potential B-cell growth factor. Several lines of evidence support this possibility. Insulin treatment has been shown to increase the regenerative activity of $\mathrm{B}$-cells in rodent models of diabetes induced by alloxan or streptozotocin (McEvoy, Schmitt et al. 1978; Movassat, Saulnier et al. 1997). A role of insulin as a growth factor is also supported by studies in B-cell-specific IR knockout (BIRKO) mice, which display an age-dependent decrease in B-cell mass (Kulkarni, Bruning et al. 1999), and by reports that MIN6 B-cells treated with IR siRNA leads to altered expression of cell cycle protein and proliferation (Ohsugi, Cras-Meneur et al. 2005). Importance of insulin and its signaling cascade in controlling B-cell mass in the context of diabetes is also supported by recent studies of mouse with knockout of some of insulin signaling components. Disruption of IRS-2, but not IRS-1, in mice leads to overt diabetes because of insufficient B-cell compensation for whole body insulin resistance (Araki, Lipes et al. 1994; Tamemoto, Kadowaki et al. 1994; Withers, Gutierrez et al. 1998; Kubota, Tobe et al. 2000). Other insulin signaling components, such as p70 S6 kinase (Pende,
Kozma et al. 2000), Akt1/PKBB (Tuttle, Gill et al. 2001), Foxo1 (Nakae, Biggs et al. 2002), 3phosphoinositide-dependent protein kinase 1 (PDK1) (Hashimoto, Kido et al. 2006), that are shown to be involved in B-cell growth by knockout or transgenic approaches, also support the importance of insulin signaling in B-cell growth in vivo. Together, these studies make a strong case for insulin as a direct or permissive factor in B-cell growth.

Several other proteins in the insulin/IGF-I signaling pathway have been linked to B-cell growth and survival responses. Notably, the transcription factor FoxO1 has been linked to the pancreatic homeodomain protein PDX-1, which was originally described to be critical only for embryonic pancreatic cell proliferation. The homeodomain protein has now been shown to be required for islet compensatory responses to insulin resistance indicating an important role in post-developmental states of B-cell growth (Kulkarni, Jhala et al. 2004).

Glucose (Bonner-Weir, Deery et al. 1989) has been shown to increase B-cell mass in rodent models, however, it is possible that the effects of glucose are mediated by secreted insulin acting in an autocrine manner (Martinez, Cras-Meneur, et al, 2006). Other mechanisms that have been proposed to contribute to regeneration of $\mathrm{B}$-cells are transdifferentiation from pancreas acinar cells or hepatocytes (Ferber, Halkin et al. 2000; Kojima, Fujimiya et al. 2003; Song, Ko et al. 2004). Whether one or all of these mechanisms are involved in islet compensatory response to insulin resistance in humans is not known.

Among pharmacological agents, the observation that sulfornylureas (SUs), widely used oral hypoglycemic agents, can induce apoptosis in rodent B-cells or cultured human islets (Efanova, Zaitsev et al. 1998; Maedler, Carr et al. 2005) has prompted intense interest in the development of therapeutic agents that preserve or restore functional B-cell mass. Several agents with the potential to inhibit B-cell apoptosis and/or increase B-cell mass have been identified in pre-clinical studies (Baggio and Drucker 2006).

In addition to GLP-1 analogs (discussed above), the thiazolidinediones (TZDs) have also been suggested to promote B-cell survival (Finegood, McArthur et al. 2001; Ishida, Takizawa et al. 2004; Kawasaki, Matsuda et al. 2005). Although it remains unclear if TZDs affect Bcell mass by direct or indirect mechanisms, it might be one attractive anti-diabetic agent that is potentially suitable for obese diabetic patients with insulin resistance, that can both improve insulin sensitivity and preserve B-cell mass. 


\section{References}

Araki, E., M. A. Lipes, et al. (1994). "Alternative pathway of insulin signalling in mice with targeted disruption of the IRS-1 gene." Nature 372(6502): 186-90.

Baggio, L. L. and D. J. Drucker (2006). "Therapeutic approaches to preserve islet mass in type 2 diabetes.” Annu Rev Med 57: 265 81.

Bonner-Weir, S., D. Deery, et al. (1989). "Compensatory growth of pancreatic beta-cells in adult rats after short-term glucose infusion." Diabetes 38(1): 49-53.

Bonner-Weir, S., M. Taneja, et al. (2000). "In vitro cultivation of human islets from expanded ductal tissue." Proc Natl Acad Sci U $S$ A 97(14): 7999-8004.

Brubaker, P. L. and D. J. Drucker (2004). "Minireview: Glucagon-like peptides regulate cell proliferation and apoptosis in the pancreas, gut, and central nervous system.” Endocrinology 145(6): 2653-9.

Bruning JC, Winnay JN, Bonner-Weir S, Taylor SI, Accili D, Kahn CR. (1997) Development of a novel polygenic model of NIDDM in mice heterozygous for IR and IRS-1 null alleles. Cell. 21;88(4):561-72.

Dor, Y., J. Brown, et al. (2004). "Adult pancreatic beta-cells are formed by self-duplication rather than stem-cell differentiation." Nature 429(6987): 41-6.

Efanova, I. B., S. V. Zaitsev, et al. (1998). "Glucose and tolbutamide induce apoptosis in pancreatic beta-cells. A process dependent on intracellular Ca2+ concentration.” J Biol Chem 273(50): 33501-7.

Ferber, S., A. Halkin, et al. (2000). "Pancreatic and duodenal homeobox gene 1 induces expression of insulin genes in liver and ameliorates streptozotocin-induced hyperglycemia.” Nat Med 6(5): 568-72.

Finegood, D. T., M. D. McArthur, et al. (2001). "Beta-cell mass dynamics in Zucker diabetic fatty rats. Rosiglitazone prevents the rise in net cell death." Diabetes 50(5): 1021-9.

Gershengorn, M. C., A. A. Hardikar, et al. (2004). "Epithelial-to-mesenchymal transition generates proliferative human islet precursor cells.” Science 306(5705): 2261-4.

Hashimoto, N., Y. Kido, et al. (2006). "Ablation of PDK1 in pancreatic beta cells induces diabetes as a result of loss of beta cell mass." Nat Genet 38(5): 589-93.

Ishida, H., M. Takizawa, et al. (2004). "Pioglitazone improves insulin secretory capacity and prevents the loss of beta-cell mass in obese diabetic db/db mice: Possible protection of beta cells from oxidative stress." Metabolism 53(4): 488-94.

Kahn, B. B. (1998). "Type 2 diabetes: when insulin secretion fails to compensate for insulin resistance." Cell 92(5): 593-6.

Kawasaki, F, M. Matsuda, et al. (2005). "Structural and functional analysis of pancreatic islets preserved by pioglitazone in $\mathrm{db} / \mathrm{db}$ mice.” Am J Physiol Endocrinol Metab 288(3): E510-8.

Kojima, H., M. Fujimiya, et al. (2003). "NeuroD-betacellulin gene therapy induces islet neogenesis in the liver and reverses diabetes in mice." Nat Med 9(5): 596-603.

Kubota, N., K. Tobe, et al. (2000). "Disruption of insulin receptor substrate 2 causes type 2 diabetes because of liver insulin resistance and lack of compensatory beta-cell hyperplasia." Diabetes 49(11): 1880-9.

Kulkarni, R. N. (2005). "New insights into the roles of insulin/IGF-I in the development and maintenance of beta-cell mass." Rev Endocr Metab Disord 6(3): 199-210.

Kulkarni, R. N., J. C. Bruning, et al. (1999). "Tissue-specific knockout of the insulin receptor in pancreatic beta cells creates an insulin secretory defect similar to that in type 2 diabetes." Cell 96(3): 329-39.

Kulkarni, R. N., U. S. Jhala, et al. (2004). "PDX-1 haploinsufficiency limits the compensatory islet hyperplasia that occurs in response to insulin resistance." J Clin Invest 114(6): 828-36.

Kulkarni RN, Almind K, Goren HJ,Winnay JN, Ueki K, Okada T, Kahn CR. (2003) Impact of genetic background on development of hyperinsulinemia and diabetes in insulin receptor/insulin receptor substrate-1 double heterozygous mice.

Diabetes. 52(6):1528-34.

Maedler, K., R. D. Carr, et al. (2005). "Sulfonylurea induced beta-cell apoptosis in cultured human islets."J Clin Endocrinol Metab 90(1): 501-6

Martinez SC, Cras-Meneur C, bernal-Mizrachi E, Permutt MA. (2006) Glucose Regulates Foxo1 Through Insulin Receptor Signaling in the Pancreatic Islet $\{$ beta $\}$-cell.

Diabetes. 55(6):1581-91

McEvoy, R. C., R. V. Schmitt, et al. (1978). "Syngeneic transplantation of fetal rat pancreas. I. Effect of insulin treatment of the reversal of alloxan diabetes." Diabetes 27(10): 982-7.

Movassat, J., C. Saulnier, et al. (1997). "Insulin administration enhances growth of the beta-cell mass in streptozotocin-treated newborn rats." Diabetes 46(9): 1445-52.

Nakae, J., W. H. Biggs, 3rd, et al. (2002). "Regulation of insulin action and pancreatic beta-cell function by mutated alleles of the gene encoding forkhead transcription factor Foxo1.” Nat Genet 32(2): 245-53.

Ohsugi, M., C. Cras-Meneur, et al. (2005). "Reduced expression of the insulin receptor in mouse insulinoma (MIN6) cells reveals 
multiple roles of insulin signaling in gene expression, proliferation, insulin content, and secretion." J Biol Chem 280(6): 4992-5003. Pende, M., S. C. Kozma, et al. (2000). "Hypoinsulinaemia, glucose intolerance and diminished beta-cell size in S6K1-deficient mice." Nature 408(6815): 994-7.

Pospisilik, J. A., J. Martin, et al. (2003). "Dipeptidyl peptidase IV inhibitor treatment stimulates beta-cell survival and islet neogenesis in streptozotocin-induced diabetic rats.” Diabetes 52(3): 741-50.

Rhodes, C. J. (2005). “Type 2 diabetes-a matter of beta-cell life and death?” Science 307(5708): 380-4.

Savagner, P. (2001). "Leaving the neighborhood: molecular mechanisms involved during epithelial-mesenchymal transition." Bioessays 23(10): $912-23$.

Song, K. H., S. H. Ko, et al. (2004). "In vitro transdifferentiation of adult pancreatic acinar cells into insulin-expressing cells." Biochem Biophys Res Commun 316(4): 1094-100.

Stoffers, D. A. (2004). "The development of beta-cell mass: recent progress and potential role of GLP-1." Horm Metab Res 36(1112): 811-21.

Tamemoto, H., T. Kadowaki, et al. (1994). "Insulin resistance and growth retardation in mice lacking insulin receptor substrate-1." Nature 372(6502): 182-6.

Tuttle, R. L., N. S. Gill, et al. (2001). "Regulation of pancreatic beta-cell growth and survival by the serine/threonine protein kinase Akt1/PKBalpha.” Nat Med 7(10): 1133-7.

Wideman, R. D. and T.J. Kieffer (2004). "Glucose-dependent insulinotropic polypeptide as a regulator of beta cell function and fate." Horm Metab Res 36(11-12): 782-6.

Withers, D. J., J. S. Gutierrez, et al. (1998). "Disruption of IRS-2 causes type 2 diabetes in mice.” Nature 391(6670): 900-4.

Zimmet, P., K. G. Alberti, et al. (2001). "Global and societal implications of the diabetes epidemic." Nature 414(6865): 782-7. 